01

\title{
Спектр электронов при холодной нелинейной эмиссии из металла под действием лазерного импульса
}

\author{
(C) П.А. Головинский, ${ }^{1,2}$ E.А. Михин ${ }^{1}$ \\ 1 Лаборатория фризических исследований, \\ Воронежский государственный архитектурно-строительный университет, \\ 394006 Воронеж, Россия \\ ${ }^{2}$ Московский фризико-технический институт (государственный университет), \\ 141700 Долгопрудный, Россия \\ e-mail: golovinski@bk.ru
}

(Поступило в Редакцию 25 февраля 2016 г. В окончательной редакции 13 июня 2017 г.)

\begin{abstract}
Рассмотрена нелинейная эмиссия электронов из металла под действием фемтосекундного лазерного импульса умеренной интенсивности. Построена теоретическая модель процесса на основе одномерного нестационарного уравнения Шредингера в полупространстве вакуума с заданными граничными условиями для волновой функции электрона, при решении которого применяется преобразование Лапласа. Считается, что лазерное поле слабо возмущает состояния свободных электронов в металле, описываемые в рамках теории металлов Зоммерфельда. Получен энергетический спектр вылетевших электронов и исследована его зависимость от параметров лазерного импульса. Результат расчета спектра нелинейной эмиссии электронов из вольфрамовой наноиглы под действием лазерного импульса с напряженностью $9.26 \mathrm{~V} / \mathrm{nm}$ и длительностью $6.5 \mathrm{fs}$ согласуется с экспериментальными данными.
\end{abstract}

DOI: 10.21883/JTF.2017.12.45195.1779

\section{Введение}

Внимание к изучению взаимодействия лазерного излучения с поверхностью металла в значительной мере обусловлено развитием лазерных приложений, связанных с наблюдением, управлением и диагностикой различных наноразмерных объектов [1-3]. Исследование эмиссии электронов, сопровождающей этот процесс, стало возможным практически сразу после начала широкого использования лазеров в экспериментальной физике [4]. В первых экспериментах с лазерными импульсами достаточно большой длительности нелинейные по полю эффекты не были обнаружены, поскольку возникновение электронной эмиссии вызывалось нагреванием мишени, т.е. процесс сводился к термоэмиссии [5-7]. С появлением технологии уменьшения длительности лазерных импульсов картина эмиссии существенно меняется. При длительностях в несколько пикосекунд происходит нарушение теплового равновесия между электронами и кристаллической решеткой, приводящее к увеличению тока эмиссии, и для теоретического описания экспериментальных наблюдений применимы двухтемпературные модели с разными температурами решетки и электронов [8].

При длительности лазерного импульса меньше времени электрон-фононной релаксации, которая составляет величину $\sim 1$ ps, удается избежать существенного нагрева мишени даже для сравнительно высоких лазерных интенсивностей $\sim 30 \mathrm{GW} / \mathrm{cm}^{2}[9,10]$. Современные установки позволяют получать лазерные импульсы длительностью в несколько фемтосекунд. Эмиссия электронов из металла под действием таких импульсов происходит без термализации электронов, поскольку времени для релаксации электронной компоненты недостаточно. Начальное состояние ансамбля электронов в металле не успевает заметно измениться за время действия лазерного импульса, и наблюдается значительная нелинейная эмиссия, являющаяся альтернативой холодной эмиссии электронов в электростатическом поле, которая активно исследуется для наноэмиттеров [11-13].

Описание процесса нелинейной холодной эмиссии электронов из металла в низкочастотном лазерном поле, как правило, происходит по двум возможным схемам. В основе первой лежит нестационарное уравнение Шредингера, начальное состояние электрона при этом описывается в рамках зонной модели [14], а конечное состояние строится в виде функции с волковской фазой, включающей в себя взаимодействие с электромагнитным полем [15]. Далее вычисляются матричные элементы для амплитуды перехода электрона в конечные состояния, и на их основе получается энергетический спектр. Вторая схема развивается в рамках теории зависящего от времени функционала плотности с использованием формализма Кона-Шема [16]. При этом электроны в металле считаются независимыми, а их движение происходит в некотором эффективном потенциале, создаваемом ионами кристаллической решетки при наличии обменного взаимодействия и электронной корреляции. Для расчета процесса электронной эмиссии из металла под действием фемтосекундного лазерного импульса уравнения Кона-Шема можно решить численно, например, методом Кранка-Николсона.

Вычислительные трудности, связанные с использованием обеих рассмотренных схем, снижают их привле- 
кательность. В настоящей работе построена аналитическая модель нелинейной холодной эмиссии электронов из металла под действием фемтосекундного лазерного импульса, лишенная указанного недостатка и дающая хорошее согласие с экспериментом.

\section{Математическая модель нелинейной эмиссии}

В основе классической теории Фаулера-Нордгейма (ТФН) [17] для холодной эмиссии электронов с поверхности металла под действием постоянного однородного электрического поля лежит предположение о том, что электроны зоны проводимости ведут себя подобно свободным частицам, движение которых в объеме металла ограничено силами двойного электрического слоя на границе раздела металл-вакуум. Наличие этого слоя приводит к скачку потенциальной энергии, в результате чего электрон отражается от границы, если его энергия недостаточна для преодоления потенциального барьера. Внешнее электрическое поле снижает потенциальный барьер, он приобретает конечную ширину, и возникает туннельный отрыв электронов, ведущий к холодной эмиссии.

Действие переменного электрического поля приводит к нестационарности процесса эмиссии. Для построения модели нелинейной холодной эмиссии электронов из металла под действием электромагнитного импульса ультракороткой длительности рассмотрим падение лазерного луча на плоскую металлическую поверхность в вакууме под большим углом к нормали (рис. 1,a). В этом случае имеется нормальная к поверхности составляющая электрического поля, вызывающая эмиссию электронов. Направим координатную ось $O x$ перпендикулярно поверхности, и выберем ее начало на границе раздела металл-вакуум. В духе ТФН будем считать, что потенциальная энергия электрона в металле представляет собой ступенчатую функцию переменной $x$ (рис. $1, b)$, а начальное распределение электронов по энергии соответствует статистике Ферми-Дирака.

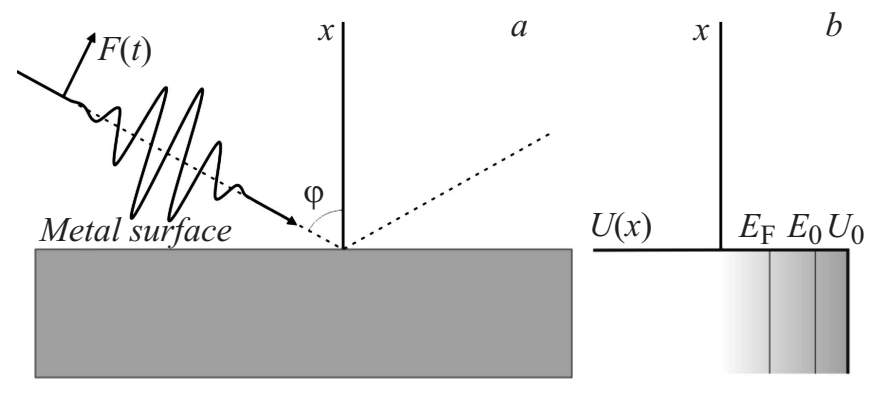

Рис. 1. Схематическое изображение падения лазерного луча на поверхность металла $(a)$ и распределения свободных электронов по энергии $(b) . U_{0}$ - энергия электрона на дне зоны проводимости, $E_{F}-$ энергия Ферми, $E_{0}-$ энергия уровня в зоне проводимости.
Характеризуя действие внешнего лазерного поля нормальной компонентой векторного потенциала $A(t)$, запишем одномерное уравнение Шредингера для электрона в вакууме в виде

$$
i \frac{\partial \psi(x, t)}{\partial t}=\frac{1}{2}\left(\hat{p}+\frac{1}{2} A(t)\right)^{2} \psi((x, t), \quad x>0 .
$$

Обозначения в уравнении (1) являются стандартными, и используется атомная система единиц: $m=\hbar=e=1$. Взаимодействие электрона с полем вне металла учтено в дипольном приближении.

До начала действия на металл поля лазерного излучения волновая функция электрона в области вакуума экспоненциально убывает с возрастанием $x$ [18]:

$$
\psi-C \exp (-\kappa x) \exp \left(-i E_{0} t\right), \quad x>0 .
$$

Здесь $E_{0}-$ энергия электрона, $\kappa=\sqrt{2\left|E_{0}\right|}$. Соответствующие граничные условия имеют вид

$$
\begin{gathered}
\psi(0, t)=\alpha(t)=C \exp \left(-i E_{0} t\right), \\
\psi_{x}^{\prime}(0, t)=\beta(t)=-\kappa C \exp \left(-i E_{0} t\right) .
\end{gathered}
$$

Штрихом в (3) обозначена производная по пространственной переменной. Рассматривая лазерный импульс, длительность которого составляет несколько фемтосекунд, а напряженность электрического поля относительно невелика, так что $F \ll \kappa\left|E_{0}\right|$, примем, что за время его действия волновые функции внутри металла меняются незначительно, и сохраняются граничные условия (3).

В рамках сформулированной модели описание нелинейной холодной эмиссии электронов сводится к решению одномерного нестационарного уравнения Шредингера (1) на полуоси $x>0$ при выполнении граничных условий (3). Такая формулировка задачи, а также отсутствие в дипольном приближении зависимости векторного потенциала $A$ от координаты $x$, обусловливают удобство использования для нахождения решения преобразования Лапласа

$$
f(s, t)=\int_{0}^{\infty} \exp (-s x) \psi(x, t) d x
$$

где $f(s, t)$ - изображение функции $\psi(x, t), s$ - комплексная переменная [19].

Вводя колебательную скорость электрона в поле волны $v(t)=A(t) / c$, запишем уравнение (1) в виде

$$
i \frac{\partial \psi}{\partial t}=-\frac{1}{2} \frac{\partial^{2} \psi}{\partial x^{2}}-i v(t) \frac{\partial \psi}{\partial x}+\frac{v^{2}(t)}{2} \psi
$$

Применив к (5) преобразование (4), получим в пространстве изображений уравнение

$$
\begin{aligned}
i \frac{\partial f(s, t)}{\partial t}= & \frac{1}{2}(-i s+v(t))^{2} f(s, t) \\
& +\left\{s \frac{\alpha(t)}{2}+\frac{\beta(t)}{2}+i v(t) \alpha(t)\right\} .
\end{aligned}
$$


Решение уравнения (6) записывается в квадратурах через классическое смещение электрона в поле волны

$$
a\left(t, t_{1}\right)=\int_{t_{1}}^{t} v\left(t_{2}\right) d t_{2}
$$

и интеграл от его кинетической энергии

$$
S\left(t, t_{1}\right)=\int_{t_{1}}^{t} \frac{v^{2}\left(t_{2}\right)}{2} d t_{2}
$$

в виде

$$
\begin{aligned}
& f(s, t)=-i \int_{0}^{t}\left(s \frac{\alpha\left(t_{21}\right)}{2}+\frac{\beta\left(t_{1}\right)}{2}+i v\left(t_{1}\right) \alpha\left(t_{1}\right)\right) \\
& \quad \times \exp \left\{i\left(\frac{s^{2}\left(t-t_{1}\right)}{2}+i s \alpha\left(t, t_{1}\right)-S\left(t, t_{1}\right)\right)\right\} d t_{1} .
\end{aligned}
$$

Замена $s=i p$ в выражении (9) дает решение уравнения Шредингера в импульсном представлении, и нахождение распределения вылетевших электронов по импульсу, соответствующее моменту окончания действия лазерного поля $T$, сводится к вычислению интеграла

$$
\begin{aligned}
& f(p, T)=-i C \int_{0}^{T}\left(\frac{i p}{2}-\frac{\kappa}{2}+i v\left(t_{1}\right)\right) \exp \left(-i E_{0} t_{1}\right) \\
& \quad \times \exp \left\{i\left(-\frac{p^{2}\left(T-t_{1}\right)}{2}-p a\left(T, t_{1}\right)-S\left(T, t_{1}\right)\right)\right\} d t_{1} .
\end{aligned}
$$

Вычислить аналитически интеграл в уравнении (10) в общем случае не удается, а прямое использование численных методов затрудняют быстрые осцилляции подынтегральной функции. В этом случае оправданным является применение метода перевала [20], учитывающего наибольший вклад в интеграл области вблизи стационарных точек, положение которых определяется из решения уравнения

$$
\frac{\partial}{\partial t_{1}}\left(-\frac{p^{2}\left(T-t_{1}\right)}{2}-p a\left(T, t_{1}\right)-S\left(T, t_{1}\right)\right)=0 .
$$

Аналогичное приближение использовано в теории многофотонной ионизации Келдыша [21,22]. Его применимость не ограничена конкретными формами лазерных импульсов, за исключением требования малости несущей частоты $\omega$ по сравнению с пороговой частотой однофотонной ионизации: $\left|E_{0}\right| / \omega \gg 1$. Уравнение (11) для отыскания стационарных точек решается численно, после чего методом стационарной фазы находится первое приближение для функции $f(p, T)$. Полученная зависимость уточняется путем численного решения дифференциального уравнения (6) с использованием неявного метода Адамса-Мултона [23].
Функция $f(p, t)$ позволяет записать выражение для энергетического распределения электронов в виде

$$
\frac{d N_{e}}{d E}=\frac{|f(\sqrt{2 E}, T)|_{T=\infty}^{2}}{\sqrt{2 E}}
$$

Выражение (12) описывает спектр вылетевших электронов, имеющих в начальный момент времени фиксированную энергию $E_{0}$. Для получения полного спектра следует учесть, что электроны в металле распределены по начальной энергии. Согласно теории металлов Зоммерфельда, число электронных состояний в единице объема металла, приходящихся на единичный интервал энергии, определяется уравнением

$$
\frac{d n_{e}}{d E_{0}}=\frac{1}{\pi^{2}} \frac{\sqrt{2\left(E_{0}-U_{0}\right)}}{1+\exp \left(\frac{E_{0}-E_{F}}{k \theta}\right)}
$$

где $\theta$ - температура металла, $k$ - постоянная Больцмана. Использование в уравнении (13) вместо химического потенциала энергии Ферми оправдано при комнатной температуре с погрешностью $\sim 10^{-3} \mathrm{eV}$.

Произведя интегрирование по начальным электронным состояниям с учетом (13), получим итоговую формулу, определяющую энергетический спектр вылетевших электронов

$$
\begin{aligned}
\frac{d N_{e}}{d E}= & \frac{1}{\pi^{2}} \int_{U_{0}}^{E_{F}} \sqrt{\frac{E_{0}-U_{0}}{E}}\left(1+\exp \left(\frac{E_{0}-E_{F}}{k \theta}\right)\right)^{-1} \\
& \times\left|f_{E_{0}}(\sqrt{2 E}, T)\right|_{T=\infty}^{2} d E_{0} .
\end{aligned}
$$

Значение $\left|E_{F}\right|$ совпадает с величиной работы выхода электрона из металла, а $U_{0}=E_{F}-\pi^{1 / 3}\left(3 n_{0} / 8\right)^{2 / 3}\left(n_{0}-\right.$ концентрация свободных электронов в металле). Основной вклад в интеграл (14) дает область вблизи энергии $E_{F}$ из-за быстрого уменьшения величины $\left|f_{E_{0}}(\sqrt{2 E}, T)\right|_{T=\infty}^{2}$ для глубоких начальных электронных уровней.

\section{Влияние параметров лазерного импульса на спектр электронов}

Конкретный вид энергетического спектра электронов, образующихся в результате нелинейной холодной эмиссии из металла, зависит от параметров лазерного импульса. Будем считать, что изменение напряженности электрического поля во времени определяется выражением

$$
F(t)=F_{0} \exp \left(-\frac{t^{2}}{\tau^{2}}\right) \cos (\omega t),
$$

где $F_{0}$ - амплитуда напряженности, $\omega-$ частота несущей лазерного импульса, $\tau$ - параметр длительности импульса. 


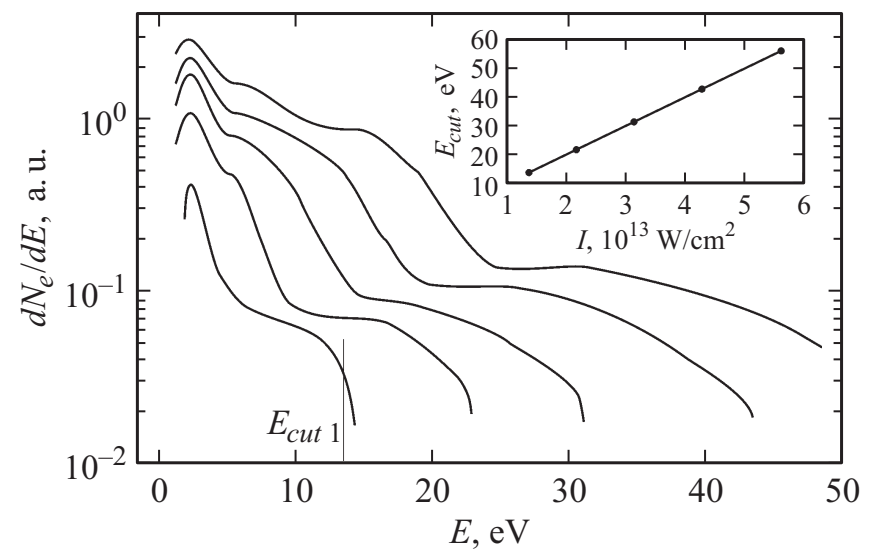

Pис. 2. Энергетический спектр вылетевших электронов. Кривым снизу вверх соответствуют значения амплитуды напряженности лазерного поля $F_{0}=10,12,15,18,20 \mathrm{~V} / \mathrm{nm}$ при $\lambda=800 \mathrm{~nm}, \tau=7 \mathrm{fs}$.

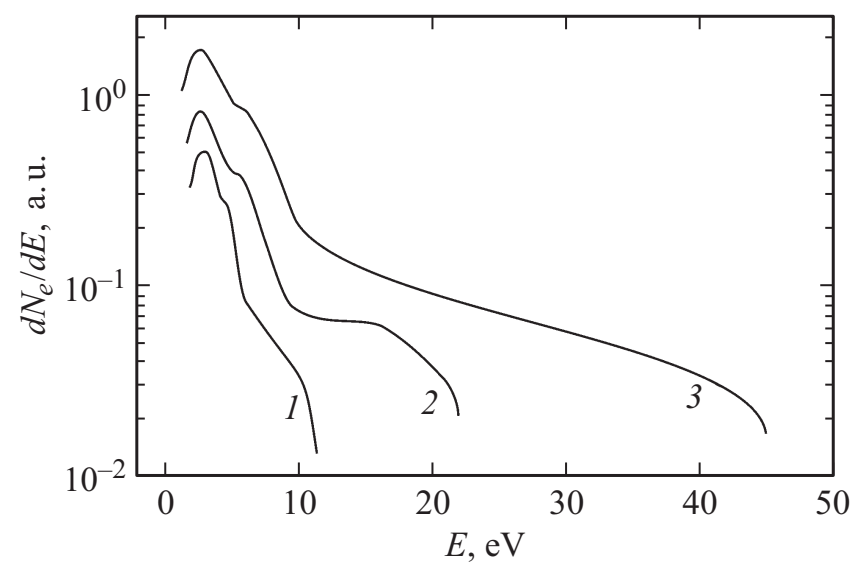

Рис. 3. Энергетический спектр вылетевших электронов. Кривая 1 соответствует значению $\lambda=600,2-800,3-$ $1200 \mathrm{~nm}$. Амплитуда поля лазерного импульса $F_{0}=12 \mathrm{~V} / \mathrm{nm}$, длительность $\tau=7 \mathrm{fs}$.

В качестве примера была рассмотрена мишень в виде пластины из золота с работой выхода $4.83 \mathrm{eV}$ [24]. На рис. 2 представлены результаты расчетов влияния амплитудного значения напряженности поля на энергетический спектр вылетевших электронов для значений $F_{0}$, лежащих в интервале $10-20 \mathrm{~V} / \mathrm{nm}$. Полученные кривые имеют характерный максимум вблизи однофотонного порога ионизации электрона из металла, вслед за которым расположен участок плато. Затем при достижении энергии отсечки электронов $E_{c u t}$, наблюдается резкий спад интенсивности спектра. С ростом амплитуды напряженности лазерного поля при прочих фиксированных параметрах происходит увеличение энергии отсечки и общего числа эмитированных электронов. Показанная на вставке рис. 2 зависимость $E_{c u t}$ от пиковой интенсивности лазерного поля свидетельствует о наличии линейной связи между этими величинами. Угол наклона прямой линии к оси абсцисс равен $41.5 \mathrm{eV} / 4.2 \cdot 10^{13} \mathrm{~W} / \mathrm{cm}^{2}$.

Энергетический спектр электронов зависит от несущей длины волны лазерного излучения $\lambda$. Как видно из результатов расчетов, приведенных на рис. 3 , при увеличении длины волны участок плато в спектре становится шире. Рост энергии отсечки пропорционален $\lambda^{2}$ в соответствии с изменением колебательной энергии электрона в поле волны. Так, для кривых 1 и 3 на рис. 3 значения длин волн отличаются в 2 раза, а $E_{\text {cut }}-$ в 4 раза.

Для оценки влияния длительности лазерного импульса на эмиссию рассчитан энергетический спектр электронов при различных значениях параметра $\tau$, в то время как остальные параметры импульса постоянны. Результаты расчета представлены на рис. 4. С уменьшением длительности импульса происходит уширение спектра лазерного излучения, поэтому имеется некото-

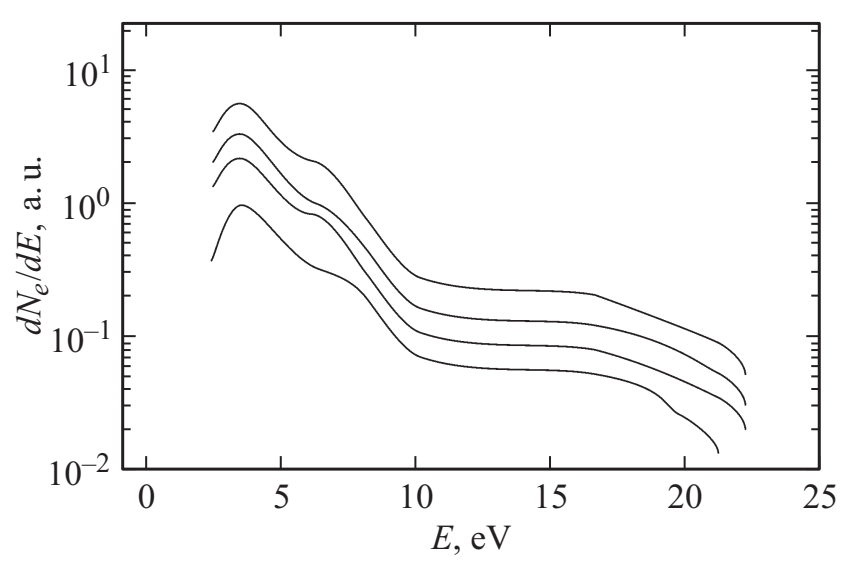

Рис. 4. Энергетический спектр вылетевших электронов. Кривым снизу вверх соответствуют значения параметра $\tau=4,8$, $16,32 \mathrm{fs}$. Напряженность поля $F_{0}=12 \mathrm{~V} / \mathrm{nm}$, несущая длина волны $\lambda=800 \mathrm{~nm}$.

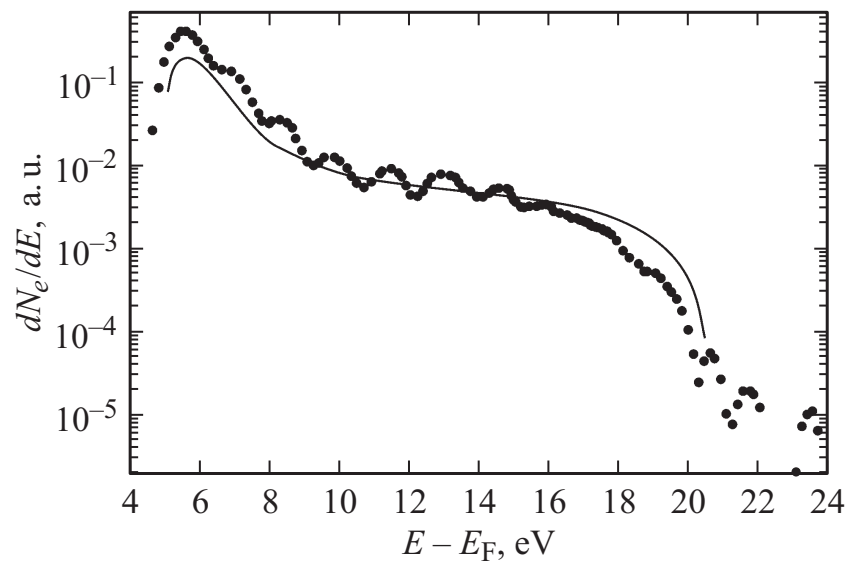

Рис. 5. Сравнение результатов расчетов спектра электронов (сплошная кривая) с экспериментальными данными [16], отмеченными точками. Значения энергии отсчитываются от уровня Ферми. 
рое отличие формы кривой, соответствующей $\tau=4 \mathrm{fs}$, вблизи энергии отсечки от форм других спектральных кривых, относящихся к более длинным импульсам. Дальнейший рост $\tau$ не меняет существенно формы спектра, а приводит главным образом к увеличению общего числа эмитированных электронов.

Варьируя значение энергии лазерного импульса, размеры, состав и геометрию облучаемой мишени, можно получать электронные пучки фемтосекундной длительности с различной энергией электронов $[1,25]$. На рис. 5 представлено сравнение результатов расчетов спектра электронов, выполненных в рамках предложенной модели, с экспериментальными данными [16]. При численном моделировании использовались следующие параметры: длина волны лазерного излучения $800 \mathrm{~nm}$, длительность лазерного импульса на половине максимума интенсивности $\sim 6.5 \mathrm{fs}(\tau \sim 13 \mathrm{fs})$, максимум напряженности лазерного поля $F_{0}=9.26 \mathrm{~V} / \mathrm{nm}$. Мишень представляла собой иглу из вольфрама с работой выхода электронов $5.22 \mathrm{eV}$ и радиусом закругления острия $\sim 8 \mathrm{~nm}$. Выбор мишени в виде наноиглы обеспечивает существенное усиление лазерного поля вблизи поверхности мишени [26,27]. Экспериментальное значение напряженности лазерного поля взято с учетом этого эффекта.

Результаты численного моделирования показывают, что предложенное теоретическое описание холодной эмиссии электронов из металлической поверхности под действием ультракороткого лазерного импульса согласуется с экспериментальными данными. Воспроизведена величина энергии отсечки, положение главного максимума в спектральном распределении электронов, а также наклон кривой в центральной части спектра. Следует отметить, что остается определенное расхождение теории и эксперимента вблизи главного максимума и вблизи энергии отсечки.

\section{Заключение}

Нелинейная холодная эмиссия электронов из металла под действием ультракороткого лазерного импульса может быть описана в рамках предложенной одномерной модели. С ее помощью проведено исследование влияния параметров лазерного импульса на энергетический спектр вылетевших электронов. Сравнение результатов теоретических расчетов с экспериментальными данными показывает, что модель хорошо воспроизводит общий вид спектра. Наличие в спектре локальных пиков, по всей видимости, связано с интерференцией между электронами, обладающими одинаковой кинетической энергией в конечном состоянии и эмитированными из разных участков поверхности. Эта модуляция спектра не воспроизводится при теоретическом описании в рамках представлений о резкой однородной границе металла. Численное моделирование рассматриваемого процесса, проделанное в [16] на основе теории зависящего от времени функционала плотности с учетом воздействия внешнего поля на электронный газ в металле, также дает некоторое расхождение с экспериментальными результатами. С хорошей точностью прописывается само положение пиков в интерференционной картине, но не их относительные величины. Для устранения отмеченного несоответствия теории и эксперимента требуется проведение дополнительных исследований.

Работа выполнена при финансовой поддержке Министерства образования и науки РФ в рамках базовой части государственного задания в сфере научной деятельности (заявка № 3.6369.2017/БЧ) и РФФИ (грант №16-3200255).

\section{Список литературы}

[1] Paarmann A., Gulde M., Muller M., Schafer S., Schweda S., Maiti M., Xu C., Hohage T., Schenk F., Ropers C., Ernstorfer R. // J. Appl. Phys. 2012. Vol. 112. P. 1131091-113109-10

[2] Germann M., Latychevskaia T., Escher C., Fink H.-W. // Phys. Rev. Lett. 2010. Vol. 104. P. 095501-1-095501-4.

[3] Chen Z.L., Zhang J., Liang T.J., Teng H., Dong Q.L., Li Y.T., Zhang J., Sheng Z.M., Zhao L.Z., Tang X.W. // At. Mol. Opt. Phys. 2004. Vol. 37. P. 539-546.

[4] Анисимов С.И., Бендерский В.А., Фаркош Д. // УФН. 1977. T. 122. C. $185-222$.

[5] Lichtman D., Ready J. F. // Phys. Rev. Lett. 1963. Vol. 10. P. 342-347.

[6] Verber C.M., Adelman A.H. // J. Appl. Phys. 1965. Vol. 36. P. $1522-1531$.

[7] Honig R.E., Woolston J.R. // Appl. Phys. Lett. 1963. Vol. 2. P. $138-143$.

[8] Li L., Zhou L., Shan Y., Zhang Y. // Int. J. Thermophys. 2015. Vol. 36. P. 183-189.

[9] Fujimoto J.G., Liu J.M., Ippen E.P. // Phys. Rev. Lett. 1984. Vol. 53. P. 1837-1844.

[10] Elsayed-Ali H.E., Norris T.B., Pessot M.A., Mourou G.A. // Phys. Rev. Lett. 1987. Vol. 58. P. 1212-1220.

[11] Лупехин С.М., Ибрагимов А.А. // ЖТФ. 2011. Т. 81. С. 109112.

[12] Васильков М.Ю., Федоров Ф.С., Ушаков Н.М., Суздальиев С.Ю. // Письма в ЖТФ. 2015. Т. 41. С. 57-63.

[13] Михайлов А.И., Кабанов В.Ф., Жуков Н.Д. // Письма в ЖТФ. 2015. Т. 41. С. 8-14.

[14] Faraggi M.N., Gravielle M.S., Silkin V.M. // Phys. Rev. A. 2004. Vol. 69. P. 042901-1-042901-8.

[15] Faraggi M.N., Gravielle M.S., Silkin V.M. // Phys. Rev. A. 2006. Vol. 73. P. 032901-1-032901-7.

[16] Krüger M., Schenk M., Hommelhoff P., Wachter G., Lemell C., Burgdorfer J. // New J. Phys. 2012. Vol. 14. P. 085019-1-085019-16.

[17] Егоров Н.В., Шешин Е.П. Автоэлектронная эмиссия. Принципы и приборы. Долгопрудный: Интеллект, 2011. $704 \mathrm{c}$.

[18] Маделунг $O$. Физика твердого тела. Локализованные состояния. М.: Наука, 1985. 184 с. 
[19] Сидоров Ю.В., Федорюк М.В., Шабунин М.И. Лекции по теории функций комплексного переменного. М.: Наука, 1989. $480 \mathrm{c}$.

[20] Федорюк М.В. Метод перевала. М.: Книжный дом ЛИБРОКОМ, 2010. 369 c.

[21] Келдыш Л.В. // ЖЭТФ. 1964. Т. 47. С. 1945-1957.

[22] Переломов А.М., Попов В.С., Терентьев М.В. // ЖЭТФ. 1966. T. 50. C. $1393-1409$.

[23] Кунин С. Вычислительная физика. М.: Мир, 1992. 522 с.

[24] Anderson P.A. // Phys. Rev. 1959. Vol. 115. P. 553-560.

[25] Головинский П.А., Михин Е.А. // Письма ЖТФ. 2013. Т. 39. Вып. 10. С. 15-21.

[26] Wachter G., Lemell C., Burgdörfer J. // Phys. Rev. B. 2012. Vol. 86. P. 035402-1-035402-5.

[27] Мануйлович E.C., Астапенко В.А., Головинский П.А. // Квантовая электроника. 2016. Т. 46. Вып. 1. С. 50-56. 